\title{
BMJ Open Physiotherapy and Anterior Resection Syndrome (PARiS) trial: feasibility study protocol
}

\author{
Anna Powell-Chandler, ${ }^{1}$ Buddug Rees, ${ }^{2}$ Carole Broad, ${ }^{2}$ Jared Torkington, ${ }^{2}$ \\ Claire O'Neill, ${ }^{3}$ Julie A Cornish, ${ }^{1,2}$ the PARiS (Physiotherapy and Anterior Resection \\ Syndrome) Trial Management Group
}

To cite: Powell-Chandler A, Rees B, Broad C, et al. Physiotherapy and Anterior Resection Syndrome (PARiS) trial: feasibility study protocol. BMJ Open 2018;8:e21855. doi:10.1136/ bmjopen-2018-021855

- Prepublication history for this paper is available online. To view these files, please visit the journal online (http://dx.doi. org/10.1136/bmjopen-2017021855).

Received 22 January 2018 Revised 8 April 2018 Accepted 10 April 2018

Check for updates

${ }^{1}$ Royal Glamorgan Hospital, Llantrisant, UK

${ }^{2}$ University Hospital of Wales, Cardiff, UK

${ }^{3}$ Swansea University, Swansea, UK

Correspondence to

Julie A Cornish;

julie.cornish@wales.nhs.uk

\section{ABSTRACT}

Introduction Rectal cancer affects more than 600 patients per year in Wales, with a 5-year survival rate of around $60 \%$. A recent report demonstrated that $19 \%$ of patients with bowel cancer had difficulty controlling their bowels after surgery, and these patients were twice as likely to report lower quality of life than those who had control. Nearly all patients will experience bowel dysfunction initially following surgery and up to $25 \%$ will experience severe bowel dysfunction on a long-term basis. The aim of this study is to test the feasibility of introducing a simple intervention in an attempt to improve bowel function following surgery for rectal cancer. We propose the introduction of an educational session from specialist nurses and physiotherapists prior to surgery and a subsequent physiotherapy programme for 3 months to teach patients how to strengthen their pelvic floor.

Methods and analysis All patients with rectal cancer planned to receive an anterior resection will be approached for the study. The study will take place in three centres over 12 months, and we expect to recruit 40 patients. The primary outcome measure is the proportion of eligible patients approached who consent to and attend the educational session. The secondary outcomes include patient compliance to the pelvic floor rehabilitation programme (assessed by patient paper or electronic diary), the acceptability of the intervention to the patient (assessed using qualitative interviews) and preoperative and postoperative pelvic floor tone (assessed using the Oxford Grading System and the International Continence Society Grading System), patient bowel function and patient quality of life (assessed using validated questionnaires).

Ethics and dissemination Ethics approval was granted. This feasibility study is in progress. If patients find the intervention acceptable, the next stage would be a trial comparing outcomes after anterior resection in those who have and do not have physiotherapy.

Trial registration number ISRCTN77383505; Pre-results.

\section{INTRODUCTION}

Rectal cancer affects more than 600 patients per year in Wales, with an overall 5-year survival rate of around $60 \% .{ }^{1}$ A recent report commissioned by the Department of Health as part of the National Cancer Survivorship Initiative
Strengths and limitations of this study

- This study addresses a significant problem in aiming to improve bowel function of patients who have had rectal surgery.

- The intervention is simple, cheap and without risk of complication.

- For those patients not having neoadjuvant treatment, there is a limited time for recruitment, educational session and starting the pelvic floor rehabilitation programme prior to surgery,

- Patients may follow one of several pathways; those who have neoadjuvant treatment will have a longer preoperative period in the study. For those who have a defunctioning ileostomy, bowel continuity may not be restored by the end of the study period (3 months) preventing full assessment of some of the secondary outcomes.

demonstrated that $19 \%$ of all patients with bowel cancer had difficulty controlling their bowels after surgery, and these patients were twice as likely to report lower quality of life (QoL) than those with control. ${ }^{2}$ Specifically for rectal cancer, surgical resection with curative intent is the primary treatment for most patients. While more patients are avoiding a permanent stoma by having an anterior resection for rectal tumours, there is a tradeoff for patients in terms of their QoL due to bowel function. ${ }^{3}$

The use of a temporary ileostomy to cover a pelvic anastomosis following anterior resection for a mid to low anastomosis is now considered standard practice for most colorectal surgeons in the UK and USA. The use of a temporary ileostomy reduces the mortality associated with an anastomotic leak. ${ }^{4}$ Standard timing for reversal is considered to be 3 months; however, there is limited evidence for optimal timings. A meta-analysis of four randomised controlled trials of early ( $<14$ days) reversal of ileostomy has shown that early closure is both feasible and safe. ${ }^{5}$ 
However, in practice, reversal of ileostomy is a benign procedure without a cancer-driven target in the UK and as such may be delayed due to a variety of factors including patient recovery, postsurgical complications or chemoradiotherapy, as well as service demand pressures. Nearly all patients will experience bowel dysfunction initially following surgery and restoration of bowel continuity (if stoma reversed) for up to 12 months. However, a significant proportion of patients will experience longterm bowel dysfunction. Studies looking at long-term follow-up after anterior resection syndrome (ARS) (1-13 years) have reported rates of major bowel dysfunction of $20 \%-56 \% .^{6-10}$

ARS was defined in 2012 as disordered bowel function after rectal resection, leading to a detriment in QoL. ${ }^{11}$ The aetiology of ARS is thought to be multifactorial, with the potential of sphincter injury during the construction of the anastomosis, alterations in anorectal physiology and the development of a pudendal neuropathy. ${ }^{12}$ It has been shown that the presence of a temporary ileostomy is a risk factor for bowel dysfunction following anterior resection, ${ }^{13} 14$ and there is limited evidence from small studies that prolonged time to ileostomy closure is an independent risk factor for anterior resection syndrome, with earlier closure (less than 3-6 months) being protective. ${ }^{9}{ }^{15} 16$ It may be postulated that prolonged inactivity of the pelvic floor and sphincter complex contributes to anterior resection syndrome.

Further work on functional bowel outcomes after anterior resection has demonstrated that poorer outcome is associated with low tumours, pelvic sepsis following anastomotic leak and in those patients undergoing neoadjuvant and adjuvant radiotherapy. ${ }^{17}$ The symptoms are defined as part of low anterior resection syndrome (LARS) and include: faecal incontinence, urgency and frequency. We sent an evaluation of bowel function to all patients who had had a low anterior resection for rectal cancer in the University Hospital of Wales in a 6-year period. There was an $80 \%$ response rate $(n=68)$. Thirty-eight patients $(56 \%)$ had major LARS symptoms. Risk factors for the development of LARS symptoms on regression analysis were the presence of a defunctioning ileostomy for more than 6 months and neoadjuvant chemoradiotherapy.

There have been a limited number of studies assessing the impact of pelvic floor rehabilitation (PFR) in patients following anterior resection surgery for rectal cancer. Physiotherapy interventions are non-invasive and inexpensive, require no sophisticated equipment and have hardly any adverse effects. In addition, physiotherapy does not exclude any other form of treatment. PFR is designed to strengthen these muscles over a period of time. Evidence from pelvic floor studies for urinary incontinence suggests a period of specific muscle training over several weeks is needed to gain muscle hypertrophy. ${ }^{18}$

A systematic review by Visser $e t a l^{19}$ evaluated the effectiveness of PFR in improving functional outcome. They were only able to include five studies with a total of 321 patients, of which approximately $90 \%$ had undergone pelvic floor training. ${ }^{20-24}$ The authors concluded that use of PFR was associated with improved functional outcome and QoL; however, they felt that the studies were of limited design quality. These studies looked at the use of pelvic floor physiotherapy after reversal of the stoma to improve symptoms that were already present. Maris $e t a l^{25}$ performed a systematic review of several different methods of conservative management for bowel dysfunction following rectal resection. Seven of the studies investigated the use of PFR, with two using biofeedback alone. One study compared patients who received 15 sessions of PFR after stoma closure with a case-matched control group who did not. ${ }^{24}$ Pucciani et $a l^{23}$ reported that $23 \%$ $(\mathrm{n}=21)$ patients were symptom free after multimodal rehabilitation. The incontinence score decreased significantly in patients having an anterior resection, and this was observed in both men $(\mathrm{p}<0.03)$ and women $(\mathrm{p}<0.02)$. Allgayer $e t a l^{22}$ reported a highly significant effect of shortterm and long-term training on incontinence scores. Patients who had undergone radiotherapy improved to the same level, despite having a worse baseline incontinence, greater stool frequency and higher use of antidiarrhoeal agents than non-irradiated patients. The majority of these studies involved small numbers, with even the three largest studies involving less than 100 patients. ${ }^{21-23}$

The aim of this study is to test the feasibility of introducing a simple intervention in an attempt to improve bowel function following surgery for rectal cancer. We propose the introduction of an educational session from specialist nurses and physiotherapists prior to surgery to teach patients how to strengthen their pelvic floor using this programme. The primary aim of this study is to see if the introduction of pelvic floor training is acceptable to patients and if they are able to comply with the programme at what may be a stressful time for themselves and their family.

The use of a PFR programme in patients undergoing colorectal cancer surgery is not standard practice in the UK. We hope to initiate future studies to assess the impact of introducing this intervention on bowel function and the effect on QoL for bowel cancer survivors.

\section{METHODS AND ANALYSIS \\ Study design}

This is a single-arm, non-randomised, prospective feasibility study assessing the acceptability of a physiotherapy-based intervention (PFR programme) for patients undergoing anterior resection for rectal cancer in Cwm Taf and Cardiff and Vale University Health Boards. This feasibility study is already in progress having started in Cwm Taf in April 2016 and Cardiff and Vale in September 2017. Recruitment is due to finish in November 2018.

\section{Study population}

All patients with rectal cancer planned to receive an anterior resection will be approached for the study. An anterior resection may be defined as removal of part or all of 
the rectum with an anastomosis of the two ends to allow bowel continuity.

\section{Eligibility criteria}

Inclusion criteria

- Diagnosed with rectal cancer.

- Planned anterior resection.

- Aged 18+ years of age.

- Able to provide informed consent.

- Suitable and capable of performing the PFR programme.

\section{Exclusion criteria}

- Unable to give informed consent.

- Not physically capable of performing the PFR programme.

\section{INTERVENTIONS}

\section{Educational session}

After screening and consent, patients will attend a preoperative educational session. The educational sessions will take place in small gender-segregated groups where possible, but due to recruitment timelines and patient availability, some sessions will be one-to-one. The session will take approximately an hour. Baseline information regarding the patient's demography, medical history (including bowel function), surgical history and current drug use will be collected. The patient will complete baseline QoL and bowel function questionnaires. Patients will then be provided with an overview of normal anatomy and bowel function, how it is changed by surgery, anterior resection syndrome and management and what the study involves.

\section{Qualitative interview}

A proportion of the sessions (6-10) will be observed by a qualitative researcher. Those that wish to participate in the interview with the qualitative interviewer to gather their opinions of the educational session can be interviewed after the educational session or, if preferred, the researcher can interview the patient at home at an agreed time. The interviews will be undertaken individually. We expect to interview 6-10 participants.

\section{PFR programme}

Either on the same day or at a later date, patients will attend for baseline pelvic floor examination and introduction to PFR with the physiotherapist. They will be asked to start pelvic floor exercises immediately and to continue until their operation and to recommence the exercises as soon as they are able to after their operation. Median time from baseline assessment to surgery is 2 weeks for patients not having neoadjuvant treatment. Those who receive neoadjuvant therapy or who wait longer than 4 weeks from baseline assessment to surgery will require an additional preoperative pelvic floor assessment and will be required to complete the bowel function and $\mathrm{QoL}$ questionnaires again 2 weeks before their surgery.
Patients will be asked to complete three sets of muscle exercises per day: fast pelvic floor contractions (10 repetitions three times per day), slow pelvic floor contractions (10 repetitions three times per day) and submaximal pelvic floor contraction (practice often throughout the day). Each set will take between 3 and 5 min to complete. They can be done at any time of the day, any location and performed in any position that is convenient and comfortable for the patient. Patients across sites will be shown the same educational presentation and given the same patient information/app to attempt to standardise the PFR programme.

Patients will attend two follow-up visits at 6-10 weeks and 3 months post-anterior resection for pelvic floor assessment and completion of bowel function and QoL questionnaires. At this point, patients with a temporary ileostomy may not yet have had it reversed, but they will not be required to attend any further follow-up. After 3 months, patients will no longer be required to continue the pelvic floor programme as part of the study but can continue to if they wish based on their individual experience and any benefits they may have derived.

Those that have consented to qualitative interview will be contacted from 6 weeks postsurgery to participate in a follow-up interview. Again we expect to interview 6-10 patients postoperatively on an individual basis, but these do not need to be the same patients that were interviewed preoperatively. The aim of the postoperative interview is to explore how the participant found the pelvic floor exercises, the diary or app and the pelvic floor assessments to determine whether this PFR programme is acceptable and feasible.

All patients that expressed an interest in the focus group will be asked to attend a focus group in order to comment on a physiotherapy DVD and contribute to its development.

\section{Sample size estimation}

Based on a previous service evaluation, it is anticipated that a minimum of 60 patients will have anterior resections performed at both participating sites during a period of 1 year. A minimum educational session attendance rate of $60 \%$ (36 patients) is projected. We expect to be able to recruit approximately 40 patients in 1 year. Recruitment will continue until 40 patients have attended an educational session or until the 1 year recruitment period is complete, whichever is first. All patients that have been approached for study participation will be allowed to continue, even if the 40 th patient attendance has been achieved in the meantime.

\section{OUTCOME ASSESSMENT}

\section{Primary outcome}

The primary outcome is the proportion of patients approached who consent to and attend the educational session. A screening log will be kept of all eligible patients, those that are approached to participate in the study 
and those who consent. For those that do not consent, a reason for refusal will be sought if possible and documented. An attendance log will be maintained for the educational session.

\section{Secondary outcomes}

Patients' compliance to the PFR programme will be assessed by reviewing the patient's paper diary or Squeezy app log. The Squeezy app has been designed by chartered physiotherapists specialising in Women's Health working in the National Health Service (NHS).${ }^{26}$ Squeezy has been reviewed and approved by the NHS Choices health apps library team for its clinical safety and costs $£ 2.99$ (the cost of which is covered by the grant funding). The app can be tailored to a specific exercise programme by the physiotherapists and set to remind patients when to do their exercises. It is simple to use, discreet, informative and has helpful visual and audio prompts to support the exercise programme. In addition, it maintains a prospective time record of the number of exercises completed, which can be reviewed by the physiotherapists. Patients will be given the option of recording their pelvic floor exercises with either a paper diary or Squeezy app.

The acceptability of the intervention will be assessed by interviewing a subset of the study population. A semistructured interview schedule has been created that will guide qualitative interviews but will also allow some freedom for the patient to discuss areas of importance to them. The schedules are tailored to whether the interview is posteducational session/preoperative or postoperative. Each group will include 6-10 individual interviews. The interview will be audio-recorded with the interviewee's permission.

Pelvic floor tone will be assessed by one physiotherapist at each site. To standardise the assessment, validated grading systems will be used: the Oxford Grading System and the International Continence Society (ICS) Grading System. There are several scales of manual muscle testing in use worldwide for pelvic floor muscle (PFM) strength testing. The Oxford scale was chosen for this study, as it is a commonly used scale for PFM assessment among physiotherapists in the UK. Laycock and Jerwood ${ }^{27}$ have established intratherapist reliability of this scale, tested in bent-knee lying. Intertester reliability still needs to be established where two or more clinicians are involved in pretreatment and post-treatment assessment. ${ }^{28}$ To this effect, we have decided to also record muscle strength using the ICS grade as well. This is a validated tool $^{29}$ and is used more frequently in international studies. This will not add time or additional testing for the patient but will allow an additional measure to be recorded for reliability and will aid the decision of which tool to use in future work.

Bowel function will be assessed with validated bowel function questionnaires. The LARS score is a simple five-question tool that was first created in 2012 in Denmark ${ }^{30}$ and has been validated for the English translation in 2014. The internationally validated score attempts to allow a uniform assessment of function in patients undergoing anterior resection.

The St Mark's faecal incontinence tool was created in $1998^{31}$ and is a modified version of the more commonly used Wexner score ${ }^{32}$ for faecal incontinence.

QoL will be assessed with validated questionnaires. EuroQol-5D is a generic QoL tool that generates a single index value for health status with considerable potential for use in healthcare evaluation and economic analysis. ${ }^{33}$

Cancer-specific QoL will be assessed with the European Organization for Research and Treatment of Cancer core quality of life questionnaire (EORTC QLQ-C30) (V.3.0) and the quality of life questionnaire for patients with colorectal cancer (EORTC QLQ-CR29) (V.2.1). ${ }^{34}$ These instruments are frequently used and are internationally validated.

As an adjunct to this study, a video/DVD of the educational session and physiotherapy programme will be developed. Patients who have participated in the educational session will be invited to a focus group to comment on the DVD; its content, ease of use and their thoughts on its merits versus a one-to-one or group session with a nurse/physiotherapist. The focus group will be audio-recorded with the attendees' permission.

\section{Data analysis}

The number of patients approached as per screening log and the number of patients who attended the educational session according to the attendance log will be used to calculate attendance rate.

Compliance will be derived from the percentage completion of the practice of PFM exercises from the daily summary of the diary or app. With adequate numbers, comparisons will be undertaken of compliance rates for patients using the app versus those using paper diaries.

Bowel function and QoL measured at baseline (following neoadjuvant treatment if required), 6 weeks and 3 months postsurgery will be analysed using $\chi^{2}$ test and risk factors for LARS analysed using risk regression.

Interview data content analysis will be undertaken by the lead qualitative researcher using NVivo V.10 with double coding undertaken by a member of the research team with qualitative data analysis experience. The transcripts will be systematically analysed through the following stages:

- Preliminary reading: transcripts are read and reread by the qualitative researcher.

- Initial coding: line-line coding is conducted with initial descriptive comments noted.

- Identification and development of emergent patterns (themes): initial comments are grouped taking account for commonality and divergence. Themes are clustered according to inter-relationships, connections and patterns across themes.

- Subsequent transcripts: new themes are tested against previous cases as non-recurring themes are tested against following cases. Connections across cases are noted to identify a set of super-ordinate themes for the 
participant cohort. A coding framework for emergent themes will be developed, validated and compared.

The quantitative data will inform what proportion of patients are willing to partake in the trial and their compliance to the programme. The qualitative interviews will help us to better understand reasons for patients not wanting to take part or failing to comply with the programme to help improve the programme for future patients and trials.

\section{Management and safety}

National Institute for Health and Care Excellence guidance (CG171) states that PFR is not associated with any risks of complications specific to the treatment. However, all of the patients involved in the study will be undergoing major surgery, and there are risks associated with this, so it is likely that serious adverse events will occur within the study group. It is considered unlikely that any related serious adverse events will occur from the PFR itself. Serious adverse event reporting will commence from start of PFR to 7 days after the final pelvic floor physiotherapy assessment.

\section{Patient and public involvement}

Two lay representatives sat on the Trial Development Group. They reviewed the protocol and all patient-related materials to assess appropriate terminology and to ensure we are putting the patient at the centre of our research goals. Patients will be invited to request study results if interested.

\section{ETHICS AND DISSEMINATION}

The trial will be conducted in compliance with the principles of the Declaration of Helsinki (2013) and the principles of Good Clinical Practice and in accordance with all applicable regulatory guidance.

The intervention does include an intimate examination as part of the pelvic floor assessment. Participants must agree to this separately. Those who do not agree can still take part and undergo the rest of the pelvic floor assessment. Following surgery, the rectal examination is only performed if agreed by the patient's consultant to avoid stress on the anastomosis.

The study will respect the rights of participating patients and ensure confidentiality of patient information. Interview data as well as diary data will be transcribed by a professional transcription company and will be stored on password-protected computers at the Swansea Trials Unit at Swansea University. Participants will be allocated an individual specific trial number, which will be used to identify their data. Participant's personal details will be stored separately by sites under the guidelines of the 1988 Data Protection Act and not entered onto the trial database. Personal data will not be kept for longer than is required for the purpose for which it has been acquired.

Patients undergoing surgery for colorectal cancer have an excellent support system through the specialist cancer nurses and the clinical team, as well as several charities and voluntary organisations. Should participants have additional questions about the trial, advice will be available from both within the research team and outside of the research team in the form of websites such as the NHS website's Clinical trials page: http://www.nhs.uk/Conditions/Clinical-trials/Pages/Takingpart.aspx.

Collaborators PARIS (Physiotherapy and Anterior Resection Syndrome) Trial Management Group: Julie A Cornish; Anna Powell-Chandler; Buddug Rees; Carole Broad; Jared Torkington; Claire 0'Neill; Karen Edwards; Julie Hepburn; Alison Turner; Mark Dunford.

Contributors AP-C helped revise the protocol and contributed to the feasibility trial. $B R$ and $C B$ helped design and draft the original protocol and contributed to the feasibility trial. CO helped write and design the analysis and revised the protocol. JT and JAC conceived, designed and drafted the original protocol. All authors agree to be accountable for all aspects of the work. All authors read and approved the final manuscript.

Funding This study was funded by an Innovation grant from Tenovus Cancer Care (charity number 1054015, grant number TIG2016-29).

Competing interests None declared.

Patient consent Not required.

Ethics approval Ethical approval for this study has been granted by the Wales Research Ethics Committee.

Provenance and peer review Not commissioned; externally peer reviewed.

Open access This is an open access article distributed in accordance with the Creative Commons Attribution Non Commercial (CC BY-NC 4.0) license, which permits others to distribute, remix, adapt, build upon this work non-commercially, and license their derivative works on different terms, provided the original work is properly cited and the use is non-commercial. See: http://creativecommons.org/ licenses/by-nc/4.0/

(c) Article author(s) (or their employer(s) unless otherwise stated in the text of the article) 2018. All rights reserved. No commercial use is permitted unless otherwise expressly granted.

\section{REFERENCES}

1. . Cancer Research UK. (accessed 2nd Mar 2018) http:?? cancerresearchuk.org/health-professional/cancer-statistics/statisticsby-cancer-type/bowel-cancer/incidence\#heading-Zero

2. National Cancer Survivorship Report 2013. http://www.ncsi.org.uk/ wp- content/uploads/Living-with-and-beyond-2013.pdf (Deownload 12th Dec 2015).

3. Cornish JA, Tilney HS, Heriot AG, et al. A meta-analysis of quality of life for abdominoperineal excision of rectum versus anterior resection for rectal cancer. Ann Surg Oncol 2007;14:2056-68.

4. Montedori A, Cirocchi R, Farinella E, et al. Covering ileo- or colostomy in anterior resection for rectal carcinoma. Cochrane Database Syst Rev 2010;5:CD006878.

5. Farag S, Rehman S, Sains P, et al. Early vs delayed closure of loop defunctioning ileostomy in patients undergoing distal colorectal resections: an integrated systematic review and metaanalysis of published randomized controlled trials. Colorectal Dis 2017;19:1050-7.

6. Battersby NJ, Juul T, Christensen P, et al. United Kingdom Low anterior resection syndrome study group. predicting the risk of bowel-related quality-of-life impairment after restorative resection for rectal cancer: a multicenter cross-sectional study. Dis Colon Rectum 2016;59:270-80.

7. Emmertsen KJ, Laurberg S. Rectal cancer function study group. impact of bowel dysfunction on quality of life after sphincterpreserving resection for rectal cancer. Br J Surg 2013;100:1377-87.

8. Carrillo A, Enríquez-Navascués JM, Rodríguez A, et al. Incidence and characterization of the anterior resection syndrome through the use of the LARS scale (low anterior resection score). Cir Esp 2016;94:137-43.

9. Sturiale A, Martellucci J, Zurli L, et al. Long-term functional followup after anterior rectal resection for cancer. Int $J$ Colorectal Dis 2017;32:83-8. 
10. Jimenez-Gomez LM, Espin-Basany E, Trenti L, et al. Factors associated with low anterior resection syndrome after surgical treatment of rectal cancer. Colorectal Disease 2018;20:195-200.

11. Bryant $\mathrm{CL}$, Lunniss $\mathrm{PJ}$, Knowles $\mathrm{CH}$, et al. Anterior resection syndrome. Lancet Oncol 2012;13:e403-e408.

12. Ziv Y, Zbar A, Bar-Shavit Y, et al. Low anterior resection syndrome (LARS): cause and effect and reconstructive considerations. Tech Coloproctol 2013;17:151-62.

13. Ihn MH, Kang SB, Kim DW, et al. Risk factors for bowel dysfunction after sphincter-preserving rectal cancer surgery: a prospective study using the Memorial Sloan Kettering Cancer Center bowel function instrument. Dis Colon Rectum 2014;57:958-66.

14. Siassi M, Hohenberger W, Lösel F, et al. Quality of life and patient's expectations after closure of a temporary stoma. Int $J$ Colorectal Dis 2008;23:1207-12.

15. Hughes DL, Cornish J, Morris C. Functional outcome following rectal surgery-predisposing factors for low anterior resection syndrome. Int $J$ Colorectal Dis 2017;32:691-7.

16. Walma MS, Kornmann VN, Boerma D, et al. Predictors of fecal incontinence and related quality of life after a total mesorectal excision with primary anastomosis for patients with rectal cancer. Ann Coloproctol 2015;31:23-8.

17. Peeters KC, van de Velde CJ, Leer JW, et al. Late side effects of short-course preoperative radiotherapy combined with total mesorectal excision for rectal cancer: increased bowel dysfunction in irradiated patients--a Dutch colorectal cancer group study. J Clin Oncol 2005;23:6199-206.

18. Hagen S, Stark D, Glazener C, et al. Individualised pelvic floor muscle training in women with pelvic organ prolapse (POPPY): a multicentre randomised controlled trial. Lancet 2014;383:796-806.

19. Visser WS, Te Riele WW, Boerma D, et al. Pelvic floor rehabilitation to improve functional outcome after a low anterior resection: a systematic review. Ann Coloproctol 2014;30:109-14.

20. Liu CH, Chen $\mathrm{CH}$, Lee JC. Rehabilitation exercise on the quality of life in anal sphincter-preserving surgery. Hepatogastroenterology 2011;58:1461-5.

21. Kim KH, Yu CS, Yoon YS, et al. Effectiveness of biofeedback therapy in the treatment of anterior resection syndrome after rectal cancer surgery. Dis Colon Rectum 2011;54:1107-13.
22. Allgayer H, Dietrich CF, Rohde W, et al. Prospective comparison of short- and long-term effects of pelvic floor exercise/biofeedback training in patients with fecal incontinence after surgery plus irradiation versus surgery alone for colorectal cancer: clinical, functional and endoscopic/endosonographic findings. Scand $J$ Gastroenterol 2005;40:1168-75.

23. Pucciani F, Ringressi MN, Redditi S, et al. Rehabilitation of fecal incontinence after sphincter-saving surgery for rectal cancer: encouraging results. Dis Colon Rectum 2008;51:1552-8.

24. Laforest A, Bretagnol F, Mouazan AS, et al. Functional disorders after rectal cancer resection: does a rehabilitation programme improve anal continence and quality of life? Colorectal Dis 2012;14:1231-7.

25. Maris A, Devreese AM, D'Hoore A, et al. Treatment options to improve anorectal function following rectal resection: a systematic review. Colorectal Dis 2013;15:e67-e78.

26. Ryan B. Nursing Standard. 28, 30, 33-33. 2014. https://itunes.apple. $\mathrm{com} / \mathrm{gb} / \mathrm{app} / \mathrm{squeezy}-\mathrm{nhs}$ - physiotherapy/id700740791? $\mathrm{mt}=8$

27. Laycock J, Jerwood D. Pelvic floor muscle assessment: the perfect scheme. Physiotherapy 2001;87:631-42.

28. Jeyaseelan S, Haslam J, Winstanley J, et al. Digital vaginal assessment: an inter-tester reliability study. Physiotherapy 2001;87:243-50.

29. Messelink B, et al. The standardization of terminology of pelvic floor muscle function and dysfunction. ICS Pelvic Floor Clinical Assessment Group 2005.

30. Emmertsen KJ, Laurberg S. Low anterior resection syndrome score: development and validation of a symptom-based scoring system for bowel dysfunction after low anterior resection for rectal cancer. Ann Surg 2012;255:922-8.

31. Vaizey CJ, Carapeti E, Cahill JA, et al. Prospective comparison of faecal incontinence grading systems. Gut 1999;44:77-80.

32. Jorge JM, Wexner SD. Etiology and management of fecal incontinence. Dis Colon Rectum 1993;36:77-97.

33. Brazier J, Jones N, Kind P. Testing the validity of the Eurogol and comparing it with the SF-36 health survey questionnaire. Qual Life Res 1993;2:169-80.

34. Aaronson NK, Ahmedzai S, Bergman B, et al. The European organization for research and treatment of cancer QLQ-C30: a quality-of-life instrument for use in international clinical trials in oncology. J Natl Cancer Inst 1993;85:365-76. 